\title{
Synovial Chondromatosis of the Suprapatellar Pouch of the Knee in a Patient with Systemic Lupus Erythematosus
}

YASUHIRO SUYAMA, MD, Division of Rheumatology, JR Tokyo General Hospital, Tokyo, Japan; SHUICHI NAKAYAMA, MD, Department of Orthopedic Surgery, JR Tokyo General Hospital, Tokyo, Japan; KIYOFUMI HAGIWARA, MD, PhD, Division of Rheumatology, JR Tokyo General Hospital, Tokyo, Japan. Address correspondence to Dr. Y. Suyama, Division of Rheumatology, JR Tokyo General Hospital, 2-1-3 Yoyogi Shibuya-ku, Tokyo 151-8528, Japan. E-mail: y-suyama@jreast.co.jp. J Rheumatol 2016;43:948; doi:10.3899/jrheum.151396

Synovial chondromatosis is an uncommon benign neoplastic disease that mainly affects men in the third to fifth decades of life. Secondary synovial chondromatosis is considered to be associated with slowly progressive degenerative joint disease, including trauma and neurological disease ${ }^{1}$. Systemic lupus erythematosus (SLE) is not related to the etiological factor.

A 75-year-old woman with a history of SLE and schizophrenia presented with a complaint of right knee pain for 3 years. There was no history of trauma. Physical examination revealed tenderness and edema over the right knee, limited range of motion in the knee, and a bony nodule palpated under the suprapatellar region. Synovial fluid analysis was negative for crystals, malignant cells, and bacteria. Radiographs of the right knee revealed multiple ossified nodules in both the suprapatellar pouch and joint space of the knee (Figure 1A). The patient was diagnosed with synovial chondromatosis and underwent open synovectomy. The patient was found to have a thickened synovium containing smaller calcific bodies within the suprapatellar pouch, which was extending into the adjacent knee joint space. An area of smaller nodules that was coexisting with multiple smaller loose bodies and the synovial tissue were removed (Figure 1B).

The characteristic loose bodies develop as a result of the proliferation of the synovial membrane. The fragments may break off from the synovial surface into the joint space, which may lead to pain, swelling, and locking of the joint. It is usually monoarticular, and the primary joints affected are the knee, hip, elbow, and humeral joint ${ }^{2}$. Extraarticular sites arising in the synovium around the tendons or bursa, and a giant solitary synovial osteochondromatosis have also been described $^{1}$. Complete synovectomy and removal of the loose bodies are recommended for pain relief and functional movement recovery ${ }^{2}$.
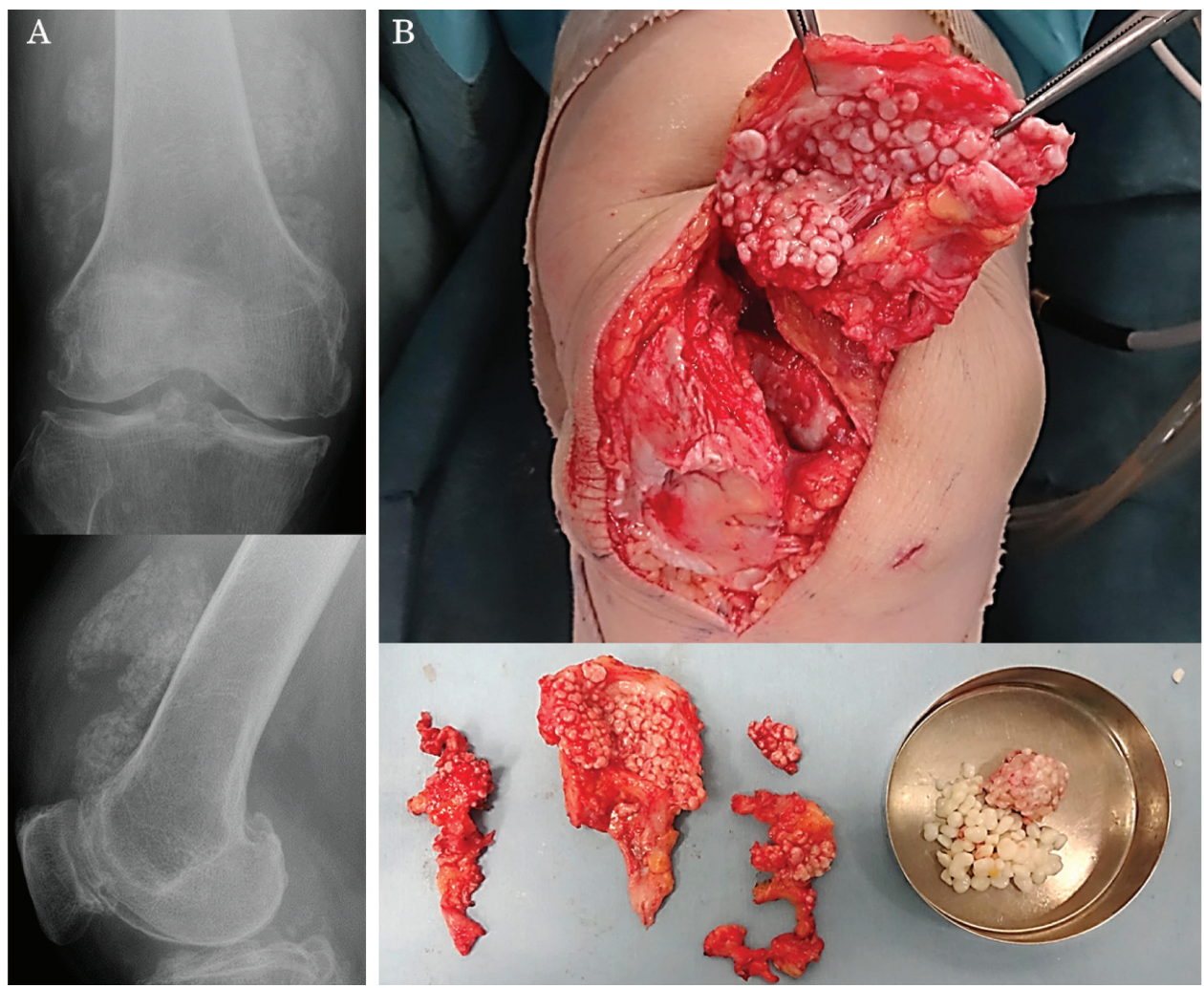

Figure 1. A. Anteroposterior and lateral radiographs of the right knee revealed multiple ossified nodules in both the suprapatellar pouch and joint space of the knee. B. Open synovectomy revealed a thickened synovium containing smaller calcific bodies within the suprapatellar pouch, which was extending into the adjacent knee joint space.

\section{REFERENCES}

1. Edeiken J, Edeiken BS, Ayala AG, Raymond AK, Murray JA, Guo SQ. Giant solitary synovial chondromatosis. Skeletal Radiol 1994;23:23-9.

2. McKenzie G, Raby N, Ritchie D. A pictorial review of primary synovial osteochondromatosis. Eur Radiol 2008;18:2662-9. 